\title{
Exposición prenatal a los plaguicidas organoclorados y criptorquidia
}

\author{
Prenatal exposure to organochlorine pesticides and cryptorchidism
}

\author{
Lília Patrícia Bustamante M ontes ${ }^{1}$ \\ Stefan Waliszewski ${ }^{2}$ \\ María Hernández-Valero ${ }^{3}$ \\ Luz Sanín-Aguirre ${ }^{4}$ \\ Rosa M aria Infanzón-Ruiz ${ }^{2}$ \\ Arlette García J añas ${ }^{2}$
}

\footnotetext{
${ }^{1}$ Facultad deM edicina, Universidad Autónoma del Estado de México. Paseo Tollocan esquina Jesus Carranzas/n. 50180Toluca Estado deM éxico. patriciiab@yahoo.com.mx ${ }^{2}$ Universidad Veracruzana.

${ }^{3}$ The University of Texas $M$. D. Anderson Cancer Center. ${ }^{4}$ Universidad Autónoma de Chihuahua.
}

Abstract Fetuses and children are more susceptible to the effects of environmental toxins. The objective of this article is to determine the levels of organochlorine pesticides ( $\mathrm{HCB}, \mathrm{B}-\mathrm{HCH}$, pp'DDT, op'DDT and pp'DDE) in the serum lipids of mothers of newborns with cryptorchidism and compare the levels to a control group of mothers of newborns with descended testicles. The cases were composed of newborns with cryptorchidism $(n=41)$, and the controls ( $n=41)$ newborns with descended testicles. Blood samplesfrom both groups of mothers were used to determinetheorganochlorinepesticidelevels. Cryptorchidism was diagnosed at birth by a neonatologist. The results showed that the organochlorine pesticide residues were found in the serum lipids of both groups of mothers. The median serum lipid levels (mgkg- 1 lipidbased) were statistically higher for the metabolites pp'DDT (0.464 vs. 0.269) and B-HCH (0.263 vs. 0.192 ) in the cryptorchidism group compared to the control group $(p<0.01)$. It could be concluded that the levels of the metabolites pp'DDT and $\beta$ $\mathrm{HCH}$ arehigher among mothers of newborns with cryptorchidism. It is possible that substances with anti-androgenic effects could produce endocrine disruption, such as cryptorchidism, during fetal development.

Key words Endocrine disrupters, Pre-natal exposure, Cryptorchidism, Organochlorine pesticides
Resumen Los productos en desarrollo y los niños son particularmente vulnerables a los tóxicos ambientales. N uestro objetivo es determinar los niveles de plaguicidas organoclorados ( $\mathrm{HCB}, \mathrm{B}-\mathrm{HCH}$, pp'DDT, op'DDT y pp'DDE) en lípidos séricos de madres de niños con criptorquidia y comparar los niveles con un grupo control de madres de niños con testículosdescendidos. El grupo de los casosfue constituido por recién nacidos con diagnóstico de criptorquidia ( $n=41)$. El grupo control $(n=41)$ se conformó por niños con testículos descendidos. A lasmadres deambos gruposseles determinaron los niveles de plagui cidas organoclorados. La criptorquidia fue diagnosticada al nacimiento por neonatólogo. Residuos de plaguicidas organoclorados fueron encontrados en lípidos séricos deambosgrupos. Los niveles de la mediana (mgkg- 1 en base lipídica) fueron mayores para los metabolitos pp'DDT (0.464 vs. 0.269) yß-HCH (0.263vs. 0.192) en el grupo con criptorquidia comparado con el grupo control. $(p<0.01)$. Los niveles de los metabolitos pp'DDT y ß-HCH son mayores entre las madres de los recién nacidos con criptorquidia. Es posible que sustancias con efectos antiandrogénicos puedan producir disrupción endocrina y criptorquidia durante el desarrollo fetal.

Palabras clave Disruptores endocrinos, Exposición prenatal, Criptorquidia y Plaguicidas organoclorados 


\section{Introducción}

Los estudios epidemiológicos de losúltimos años revelan que los casos de desórdenes reproductivos en el hombre se han incrementado, lo quese expresa como disminución del número deespermatozoides, cáncer testicular y defectos congénitos como la criptorquidia e hipospadias'. Las anomalías pueden relacionarse entresí y tener su origen común durante el desarrollo.

La hipótesis más fuerte relacionada con el decremento en la salud reproductiva masculina tiene que ver con la exposición a químicos ambientales con actividad estrogénica y/o antiandrogénica, estas sustancias llegan al hombre a través de vapores, alimentos, agua ${ }^{2}$. Entre los contaminantes se enlistan los plaguicidas organoclorados, bifenilos policlorados (PCBs), dioxinas, detergentes y plastificantes ${ }^{3-6}$. Los mecanismos de su acción farmacológica no están bien definidos, pero por su actividad hormonal 0 antihormonal consiste en: modular los efectos de las enzimas que controlan el metabolismo de las hormonas sexuales, influir directamente en las glándulas productoras de hormonas o formar mecanismos de retroalimentación indirecta que afectan a los niveles de estrógenos $\mathrm{s}^{7,8}$.

Las primeras doce semanas de gestación son críticas en la diferenciación sexual de los genitales externos del hombre y corresponde además al período de mayor susceptibilidad a los efectos de compuestos que influyen en el balance hormonal. El metabolito de la testosterona, la dihidrotestosterona induce el desarrollo de la próstata y los genitales externos masculinos. Durante la diferenciación sexual, la exposición del feto a niveles residuales de xenoestrógenos puede inducir a anomalías irreversibles. Duranteel segundo y tercer trimestre, continúa el crecimiento de estructuras dependientes de los andrógenos. Los compuestos químicos queimitan o actúan como hormonas esteroides pueden interferir en grados diferentes a la organogénesis, cuya acción depende desu concentración, tiempo de exposición y grado de eficiencia farmacológica9,10.

La criptorquidia, es la ausencia de uno o ambos testículos en las bolsas escrotales e indica, que el testículo queda detenido durante su descenso desde el retroperitoneo hasta la raíz del escroto en algún punto de su trayecto antes de alcanzar su ubicación definitiva en la bolsa escrotal. El descenso de los testículos es determinado por los andrógenos ${ }^{11}$. La importancia de la criptorquidia para la salud pública radica en que es uno de los pocos factores de riesgo bien esta- blecidos en la etiología del cáncer testicular, tumor más común en los hombres jóvenes ${ }^{12}$.

Algunos compuestos químicos sintéticos con estructura molecular diferente a las hormonas esteroides, pueden interferir una vez incorporados al organismo en el mensaje transmitido por las hormonas endógenas ${ }^{13}$. Los plaguicidas organoclorados más comunes incluyen: DDT, Metoxicloro, Clordano, Heptacloro, Aldrin, Dieldrin, Hexaclorobenceno, Lindano. El uso propagado del DDT originó sus residuos en el ambientey su propagación alrededor del mundo. En M éxico hasta el año 1999 fue utilizado en gran escala durante las acciones de salud pública en el combate del paludismo. El plaguicida pp'DDT, especialmente su isómero op'DDT revela actividad estrogénica, mientras quesu metabolito mas persistente el $\mathrm{pp}$ 'DDE posee actividad antiandrogé nica ${ }^{14,15}$. Aunque estos compuestos no presentan similitudes en la estructura química con las hormonas esteroídes naturales, son capaces de reaccionar con los receptores hormonales o bloquear los receptores androgénicos ${ }^{16}$. Se ha logrado un consenso relacionado con la capacidad estrogé nica 0 antiandrogénica de los plaguicidas organoclorados, mientras que todavía existe discusión sobre su capacidad de influir en el desarrollo del cáncer mamario y en las alteraciones reproductoras masculinas.

El objetivo de este estudio fue determinar los niveles de plaguicidas organoclorados persistentes (el Hexaclorobenceno (HCB) el isómero beta dehexaclorociclohexano $(\beta-\mathrm{HCH})$, el para, para' diclorodifenildicloroeteno ( $p p^{\prime} D D E$ ), el isómero orto, para' diclorodifeniltricloroetano (op'DDT), el isómero para, para' diclorodifeniltricloroetano ( $p p^{\prime} D D T$ ) en lípidos séricos de madres de niños con testículos no descendidos (criptorquidia) y comparar los niveles con un grupo control de madres de niños con testículos descendidos.

\section{Material y métodos}

Diseño

Para lograr el objetivo planteado se estudiaron binomios de madres-hijos varones y se formaron dos grupos, que fue conformándose el mismo día en que ocurrían los nacimientos. El grupo de los casos fue constituido por recién nacidos con diagnóstico de criptorquidia $(n=41)$. El grupo control $(n=41)$ se conformó por niños con testículos descendidos. A las madres de ambos grupos de niños se invitó a participar en el estu- 
dio. Una vez que aceptaron mediante la firma de la carta de consentimiento informado les fueron tomadas una muestra de sangre para determinar los niveles de plaguicidas organoclorados. Como se mencionó previamente, los controles fueron seleccionados en la misma fecha en que los casos nacieron. Para determinar si el niño presentaba o no criptorquidia, al nacimiento todos los recién nacidos fueron explorados por pediatra neonatólogo participante del proyecto. Todos los niños nacieron a término $40 \pm 2$ semanas de gestación, sin malformaciones congénitas o complicaciones que requirieran tratamiento en terapia intermedia intensiva. Todos los niños procedías de un H ospital General que da atención a la población del Puerto deVeracruz y áreas circunvecinas. De bido a que los plaguicidas organoclorados permanecen estables a través del tiempo, la toma de la muestra después del nacimiento del niño no modifica su concentración y puede considerarse que fue la exposición duranteel embarazo.

\section{Análisis de las muestras}

Se obtuvieron aproximadamente $10 \mathrm{ml}$ de sangre venosa mediante tubo vacutainer, las muestras fueron centrifugadas para obtener el suero a 2000 revoluciones por minuto y guardadas en refrigeración hasta su procesamiento. Se identificaron y cuantificaron 5 sustancias o metabolitos: (HCB), (B-HCH), (pp'DDE), (op'DDT) y (pp'DDT). Las muestras se analizaron por cromatografía de gases con detección por captura de electrones en un cromatógrafo de gases $\mathrm{Va}$ rian modelo 3400 CX, mediante método analítico descrito por Waliszewski et al.17. Los límites de detección fueron para HCB 0.004 mgkg-1, bHCH 0.020 mgkg- 1 , pp'DDE 0.002 mgkg- 1 , op'DDT y pp'DDT 0.003 mgkg-1 La cantidad de lípidos séricos se determinó colorimétricamente.

\section{Análisis estadísticos}

Los valores de plaguicida organoclorados en las muestras del grupo control y en el grupo con criptorquidias, se calcularon utilizando estadística básica como: medias y desviación estándar $(x \pm D E)$, medianas y los rangos. Para calcular la variabilidad entre el grupo control y el grupo con criptorquidias y debido a que las concentraciones determinadas no poseen distribución normal, seaplicó la prueba M ann-Whitney para comparar la diferencia entre las medianas. Además, se calcularon las razones de momios (OR) para determinar la asociación entre los niveles de plaguicidas organoclorados en lípidos séricos y la criptorquidia. Los cálculos estadísticos se realizaron utilizando el programa estadístico M initab versión 12 .

\section{Resultados}

Un total de 82 binomios madres-hijos fueron estudiados, 41 pares del grupo de los casos y 41 pares del grupo de los controles. Los niveles de plaguicidas organoclorados determinados en 41 muestras séricas de las madres de niños sin criptorquidia control y 41 muestras séricas de madres cuyos hijos presentaron criptorquidias se presentan en la Tabla 1. Se observa que existe tanto diferencia estadísticamente significativa en las medianas del para $\beta-\mathrm{HCH}$ y del pp'DDT. La fuente de exposición a los plaguicidas organoclorados, se identifica por el cociente de las concentraciones entreel plaguicida pp'DDT y su metabolito pp'DDE (DDT/DDE). Parael grupo control el valor calculado fue promedio de 0.148 \pm 0.050 , mediana $0.121,95 \%$ IC $0.096,0.188$ y el rango interquartil 0.092. Para el grupo con estudiado con testículos no descendidos, los

Tabla 1. M ediana y rangos de las concentraciones de plaguicidas organoclorados en lípidos séricos ( $\mathrm{mg} / \mathrm{kg}$ ) de muestras de sangre de madres de niños.

\begin{tabular}{lccccccc}
\hline \multirow{2}{*}{ Plaguicidas } & \multicolumn{3}{c}{ Casos de criptorquidia } & & \multicolumn{3}{c}{ Controles } \\
\cline { 2 - 5 } \cline { 6 - 8 } & Mediana & Mínimo & Máximo & & Mediana & M ínimo & M áximo \\
\hline HCB & 0.174 & 0.026 & 0.640 & & 0.134 & 0.031 & 0.383 \\
$\beta$-HCH & 0.192 & 0.025 & 0.536 & & 0.263 & 0.094 & 0.889 \\
pp'DDE & 2.597 & 1.274 & 6.139 & & 2.219 & 0.888 & 4.608 \\
op'DDT & 0.099 & 0.020 & 0.318 & & 0.088 & 0.005 & 0.181 \\
pp'DDT & 0.464 & 0.186 & 1.277 & & 0.269 & 0.057 & 0.838 \\
$\Sigma$-DDT & 3.193 & 1.622 & 7.262 & & 2.840 & 1.221 & 5.392 \\
\hline
\end{tabular}

*Prueba M ann Whitney; **Estadisticamentesignificativa. 
val oresfueron siguientes: promedio $0.189 \pm 0.029$, mediana $0.186,95 \%$ IC $0.170,0.230$, rango interquartil 0.033 . Calculando la diferencia entre ambos grupos, al aplicar la prueba t-Student se encontró las diferencias significativas $(p<0.01)$ entrelos valores promediosy aplicando la prueba Mann-Whitney, se encontró las diferencias significativas entrelas medianas ( $p=0.056)$, (datos no mostrados en las tablas).

Para evaluar el riesgo de criptorquidias, se calculó la razón de momios para la relación de concentraciones $\mathrm{pp} \mathrm{D}^{\prime} \mathrm{DE} / \mathrm{pp} \mathrm{DDT}^{\prime}$. Los momios en esta relación revelaron el valor promedio de 1.44 y IC $95 \%(1.12,1.75)$. Estos val ores muestran una posible relación positiva entre las concentraciones del pp'DDE y el plaguicida $\mathrm{pp}^{\prime} \mathrm{DDT}$ en suero materno y presencia de criptorquidias en varones recién nacidos ( $D$ atos no mostrados en las tablas). La asociación cruda entre los niveles de pp'DDT y criptorquidia muestra en la Tabla 2 valores estadísticamente significativos con una OR de 1.79 e IC al 95\% $1.34,2.24$.

\section{Discusión}

La criptorquidia consiste en la falta de descenso de uno o ambos testículos hacia el escroto. Se reserva para aquellos testículos que están localizados en cualquier lugar del trayecto normal y que no es posible hacerlos descender.

Se sugiere que la exposición materna a los xenoestrógenos ambientales como los plaguicidas organoclorados influye el desarrollo de los genitales masculinos en el útero. Estudios realizados en animales indican la posibilidad de acción de los xenoestrógenos en las malformaciones del tracto urogenital. Por otra parte, en estu-

Tabla 2. Razón de momios (OR) de exposición a los metabolitos de plaguicidas organoclorados y posibilidad de presentar criptorquidia.

\begin{tabular}{lcl}
\hline Plaguicidas & OR & $(95 \% \mathrm{IC})$ \\
\hline HCB & $1.17^{*}$ & $(1.08,1.27)$ \\
$\beta-H C H$ & 0.93 & $(0.69,1.16)$ \\
pp'DDE & $1.10^{\prime}$ & $(1.05,1.16)$ \\
op'DDT & $1.28^{*}$ & $(1.14,1.43)$ \\
pp'DDT & $1.79^{*}$ & $(1.34,2.24)$ \\
$\Sigma$-DDT & $1.14^{*}$ & $(1.09,1.19)$ \\
\hline
\end{tabular}

*Estadisticamente significativos. dios clínicos se ha visto que al gunos niños con malformaciones del tracto urogenital presentan defectos en el metabolismo de la testosterona 0 en los receptores de la testosterona.

Los plaguicidas organoclorados permanecen por mucho tiempo en el ambienteo en los depósitos lipídicos de los organismos. Por su propiedad lipofílica, se acumulan en la grasa y biomagnifican en la cadena alimenticia. De tal forma, que la exposición a estas sustancias a lo largo de la vida deuna mujer determina el nivel acumulado en el cuerpo y, por su paso a través de la placenta, la exposición del feto desdeel momento de su concepción ${ }^{17-19}$. Esto es importante para los estudios epidemiológicos ya que permite tomar muestras únicas durante el embarazo o posterior a él sin modificación importante para medir la potencial exposición durante el embarazo.

En los últimos 40 años, varios estudios indican el aumento de incidencia de anomalías genitourinarias en el hombre, tales como criptorquidias. Estas anomalías genitourinarias pueden relacionarse y tener un origen común en la embriogénesis. Ya que se ha dado el deterioro en un periodo relativamente corto, se sospecha como promotores de estas patologías a los agentes ambientales. Esta posibilidad se ve apoyada por el hecho de que algunos contaminantes ambientales poseen actividad estrogénica 0 antiandrogé nica. Entre los contaminantes, los plaguicidas organoclorados pudieran causar este deterioro reproductivo. Los mecanismos de acción de estos compuestos no se conocen completamente, pero pueden tener una actividad antiandrogénica, modular los efectos de las enzimas que controlan el metabolismo delas hormonas sexuales, influir directamente en las glándulas productoras de hormonas o producir mecanismos de re troalimentación indirecta que afecta a los niveles de estrógenos. La mayor parte de exposición en la población general se da por inhalar sus vapores y por medio de la dieta, al consumir alimentos contaminados.

Los resultados del estudio indican la presencia de los residuos de plaguicidas organoclorados en lípidos séricos en las madres de ambos grupos. Debido a quela procedencia delas muestras fue al eatoria y que la distribución de los resultados del análisis no posee carácter normal, en la Tabla 1 se enlistaron los resultados de las medianas para compararlos entre el grupo control y el grupo con criptorquidias. Los valores (mgkg-1 en base lipídica) indican concentraciones superiores de HCB (0.174 vs. 0.134$)$, pp'DDE (2.597 vs. 2.219 ), op'DDT (0.099 vs. 0.088 ) y 
pp'DDT ( 0.464 vs. 0.269 ) en el grupo decriptorquidias y del $\beta-\mathrm{HCH}(0.263$ vs. 0.192$)$ en el grupo control. En la Tabla 1 se muestran los resultados que se obtuvieron al probar si había diferencia en las concentraciones entre ambos grupos, al aplicar la prueba de comparación entrelas me dianas ( $M$ ann Whitney) el resultado reveló diferencias significativas $(p<0.05)$ entre las concentraciones de $\beta-H C H$ y el plaguicida pp'DDT determinadas en ambos grupos.

La asociación entreuna exposición y un daño y su magnitud, se estipula calculando la razón de momios, cuyos resultados se muestran en la Tabla 2. Los momios de exposición para el plaguicida pp'DDT son mayores entre las madres de niños que padecen criptorquidia que entre las madres de niños con descen so testicular, esta asociación es estadísticamentesignificativa OR=1.79 IC $95 \%(1.34,2.24)$. Estos resultados soportan la hipótesis planteada, en el sentido de que sustancias con efectos antiandrogénicos pueden producir disrupción endocrina durante el desarrollo manifestado en este caso como criptorquidias como ya ha sido demostrado en animales y empieza a confirmarse en humanos ${ }^{19-21}$.

Variables como la edad y la escolaridad se probaron en modelos multivariados los cuales no mostraron ninguna asociación ni modificación de la medida de asociación probablemente por la poca variabilidad mostrada. Sin embargo consideramos que para futuros estudios deberán recopilarse mayor información sobre características sociodemográficas.

Considerando los riesgos a los que puede exponerse un niño con criptorquidia, incluyendo infertilidad, riesgo de exposición a agentes físicos (calor) y riesgo aumentado para cáncer ya que ha sido una de las patologías incluidas en el Ilamado síndrome de disgenesia testicular (SDT) vale la pena la profundización en este aspecto a través de programas de investigación acción que incluyan la vigilancia epidemiológica en poblaciones de riesgo y/o con antecedentes epidemiológicos de exposición.

\section{Colaboradores}

LPB Montes, S Waliszewski, M HernándezValero, L Sanín-Aguirre, RM Infanzón-Ruiz eAG Jañas participarán igualementedetodas las etapas del documento.

\section{Agradecimientos}

Este trabajo se desarrolló gracias a la contribución económica y de colaboración de los integrantes de la Red de Cuerpos A cadémicos Salud, Trabajo y Ambiente pertenecientes a la Universidad Veracruzana, Autónomas de los Estados de M éxico y de Chihuahua y con la colaboración de la Universidad de Texas M . D. Anderson Cáncer Center. 


\section{Referencias}

1. Giwereman A, Carslsen E, Keiding, Skakkebaek NE. Evidence for increasing incidence of abnormalities of de human testis: a Review. Environ Health Perspect 1993; 101(2):65-71.

2. Sharpe RM, Skakkebæak NE. M ale reproductive disorders and the role of endocrine disruption: advances in understanding and identification of areas for future research. Pure and A pplied Chemistry 2003; 75:2023-2038

3. Bhatia R, Shiau R, Petreas M, Weintraub JM, Farhang $L$, Eskenazi B. Organochlorine pesticides and male genital anomalies in the child health and development studies. . Environ $\mathrm{H}$ ealth Perspect 2005; 113(2):220-224.

4. Damgaard IN, Skakkebaek NE, Toppari J, Virtanen $H E$, Shen H, Schramm KW, Petersen JH, Jensen TK, $M$ ain KM. Persistent pesticides in human breast milk and cryptorchidism. Environ Health Perspect 2006; 114(7):1133-1138

5. Edwards TM, Moore BC, Guillette LJ, Jr. Reproductive dysgenesis in wildlife: a comparative view. Int J Androl 2006; 29(1):109-121.

6. Jobling S, Reynolds T, White R, Parker M G, Sumpter JP. A variety of environmentally persistent chemicals, including some phthalate plasticizers, are weakIy estrogenic. Environ Health Perspect 1995; 103(6):582-587.

7. Guillette LJ, Jr. Endocrine disrupting contaminants- beyond the dogma. Environ $\mathrm{H}$ ealth Perspect 2006; 114 Suppl 1:9-12.

8. Waliszewski SM, Carvajal O, Infanzón RM, Trujillo P. Xenoestrógenos ambientales y la salud. IDEAS 2004; 23:72-87.

9. Cheek AO, M CLachlan JA. Environmental hormones and the male reproductive system. J Androl 1998; 19(1):5-10

10. Greco TL, Duello TM, Gorski J. Estrogen receptors, estradiol, and diethylstilbestrol in early development: the mouse as a model for the study of estrogen receptors and estrogen sensitivity in embryonic development of male and female reproductive tracts. Endocr Rev 1993; 14(1):59-71.

11. Cavaría O. Criptorquidia. Arch Pediatr Urug 2001 72(4):286-287.

12. Hardell L, Van Bavel B, LIndström G, Ericsson M Carlberg M. In utero exposure to persist organic pollutants in relation to testicular cancer risk. Int J Androl 2005; 29:228-234.

13. M CLachlan JA, Arnold SE. Environmental estrogens. Amer Sci 1996; 84:452-461.
14. Kelce WR, Stone CR, Laws SC, Gray LE, Kemppainen JA, Wilson EM. Persistent DDT metabolite $p, p^{\prime}-D D E$ is a potent androgen receptor antagonist. Nature 1995; 375(6532):581-585.

15. Veeramachaneni DN, Palmer JS, Amann RP, Pau $K Y$. Sequelae in male rabbits following developmental exposure to $p, p^{\prime}-D D T$ or a mixture of $p, p^{\prime}$ DDT and vinclozolin: cryptorchidism, germ cell atypia, and sexual dysfunction. Reprod Toxicol 2007; 23(3):353-365.

16. Arnold SF, Collins BM, Robinson M K, Guillette LI $M$ CLachlan JA. Differential interaction of natural and synthetic estrogens with extracellular binding proteins in a yeast estrogen screen. Steroids 1996; 61:642-646.

17. Waliszewski SM, Aguirre AA, Infanzón RM, Siliceo J. Carry-over of persistent organochlorine pesticides through placenta to fetus. Salud Pública de M éxico 2000; 42(5):384-390.

18. Waliszewski SM, Aguirre AA, Infanzon RM, Silva CS, Siliceo J. Organochlorine pesticide levels in maternal adipose tissue, maternal blood serum, umbilical blood serum, and milk from inhabitants of Veracruz, M exico. Arch Environ Contam Toxicol 2001; 40(3):432-438.

19. Brucker-Davis F, Wagner-M ahler K, D elattre I, Ducot B, Ferrari $P$, Bongain A, Kurzenne JY, M as JC, Fénichel P. Cryptorchidism at birth in Nice area (France) is associated with higher prenatal exposure to PCBs and DDE, as assessed by colostrum concentrations. H um Reprod. 2008; 23(8):1708-1718.

20. Fernandez MF, Olmos B, Granada A, López-Espinosa $M J$, M olina-M olina JM, Fernandez JM, Cruz $M$, Olea-Serrano F, Olea N. Human exposure to endocrine- disrupting chemicals and prenatal risk factors for cryptorchidism and hypospadias: a nested case-control study. Environ Health Perspect 2007; 115 (Suppl) 1:8-14.

21. Mauduit C, Florin A, Amara S, Bozec A, Siddeek B, Cunha S, Meunier L, Selva J, Albert M, Vialard F, Bailly $M$, Benahmed $M$. Long-term effects of environmental endocrine disruptors on male fertility. Gynecol Obstet Fertil. 2006; 34(10):978-984.

Artigo apresentado em 29/01/2008

Aprovado em 07/04/2008

Versão final apresentada em 29/10/2008 